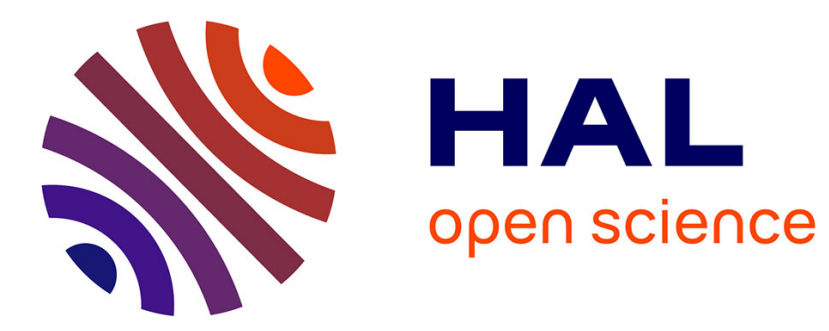

\title{
La question du genre et du nombre des autonymes en latin
}

Christian Nicolas

\section{To cite this version:}

Christian Nicolas. La question du genre et du nombre des autonymes en latin. Histoire Epistémologie Langage, 2005, 27/1 (2005): volume 27 (tome 1), p. 45-72. 10.3406/hel.2005.2049 . hal-00327492

\section{HAL Id: hal-00327492 \\ https://univ-lyon3.hal.science/hal-00327492}

Submitted on 8 Oct 2008

HAL is a multi-disciplinary open access archive for the deposit and dissemination of scientific research documents, whether they are published or not. The documents may come from teaching and research institutions in France or abroad, or from public or private research centers.
L'archive ouverte pluridisciplinaire HAL, est destinée au dépôt et à la diffusion de documents scientifiques de niveau recherche, publiés ou non, émanant des établissements d'enseignement et de recherche français ou étrangers, des laboratoires publics ou privés. 
HISTOIRE EPISTEMOLOGIE LANGAGE

(H.E.L.)

TOME XXVII, FASCICULE 1 (2005)

L'AUTONYMIE

p. $45-72$ 


\title{
La question du genre et du nombre des autonymes en latin
}

\author{
Christian NICOLAS \\ Université de Lyon, Université Jean Moulin - Lyon 3
}

L'autonyme fonctionne comme un nom. Mais, morphologiquement, il n'a pas les caractéristiques du nom : il peut être la mention d'un verbe ("Manger est un verbe du premier groupe"), d'un adjectif ("Épicène est épicène"), d'un élément plus petit que le signe (" $p$ est la seizième lettre de l'alphabet") ou plus grand ("Ah! Que ce Quoi qu'on die est d'un goût admirable!"), etc. Même lorsqu'il est la mention d'un nom, il n'a pas les caractéristiques du nom usuel qu'il mentionne (il en est, pour les logiciens, l'homonyme): en français, l'autonyme se démarque de la langue usuelle par l'emploi spécial de la détermination nominale et par la neutralisation du genre et du nombre : tout segment autonymique prend en français la catégorie non marquée sur le critère du genre et du nombre, donc l'autonyme est masculin singulier (d'où l'accord spontané dans "Moules marinières est féminin"), et il a tendance à inverser les marques de détermination, donc le nom autonyme perd son déterminant usuel ("Moules marinières..." et non pas "les Moules marinières...", dans la parenthèse précédente), alors que l'adjectif autonyme, le verbe autonyme, le phrasillon autonyme, etc., prennent facilement un déterminant ("ce Quoi qu'on die", "J'ai fort aussi l'Ingrate dans la tête", dans Les Femmes savantes). / (p. 46)

En français, le segment autonyme est donc considéré, pour la morpho-syntaxe, comme un nom masculin singulier ${ }^{1}$. On aurait tendance à croire, par analogie, que l'autonyme latin est tenu spontanément pour un nom neutre singulier. On verra ce qu'il en est ci-dessous. Mais d'ores et déjà, on peut dire que le problème syntaxique d'insertion de l'autonyme dans le tissu phrastique est un problème de translation: l'autonymie transforme en substantif logique et grammatical n'importe quel élément de l'énoncé, et pour cette opération spécifique la langue est plus ou moins bien armée. Il y a même lieu de croire que le latin et les langues flexionnelles en général, notamment celles qui sont casuelles, sont plutôt plus gênées que d'autres par cette translation, du fait que le nom a des marques spécifiques d'insertion qui ne sont pas compatibles avec n'importe quel segment : comment donner une marque par exemple d'accusatif à un autonyme non substantival ? Inversement, quand le segment autonymique est la mention d'un nom, la langue casuelle a plus de mal qu'une autre à en neutraliser les marques morpho-syntaxiques : comment décasualiser un élément qui est intrinsèquement casuel ?

\section{Vers une théorie du signe}

Avant d'aborder le traitement pratique de la question du genre et du nombre de l'autonyme en latin, nous pensons indispensable de faire un détour par la théorie. Comment les Latins se représentent-ils la dichotomie usage / mention?

À dire vrai, ils la pratiquent spontanément (comme tout locuteur, ils disposent de la fonction métalinguistique), mais ne l'ont guère théorisée. L'homonymie entre le mot en usage et le mot en mention est sporadiquement remarquée par tel lexicographe. Par exemple on peut être frappé de cette formulation étymologique de Festus ${ }^{2}$ :

Par facilité, nous appellerons "Festus" aussi bien Sextus Pompeius Festus que son abréviateur PaulDiacre, sans les différencier l'un de l'autre. Les références seront données d'après l'édition Lindsay citée en bibliographie. Le premier nombre correspond au numéro de page, le second au numéro de ligne du premier mot de la citation. Même usage pour les références faites à pour Nonius. 
FEST. 27, 5: Aues ab aduentu earum dicuntur, quod inde veniant, unde non quis suspicetur, "les oiseaux tirent leur nom de leur manière d'arriver, parce qu'ils viennent de là où on ne les attend pas".

Je ne prétends pas expliquer cette étymologie, plutôt obscure ${ }^{3}$. Je me contenterai de remarquer que, syntaxiquement, on attend aues ab suo aduentu dicuntur. Festus aperçoit-il confusément une dichotomie dans le signe, avec un versant utile en usage ("les oiseaux vont et viennent") et un versant utile en mention ("le mot oiseau a telle étymologie") ? En tout cas l'absence de réfléchi, chez un auteur qui, par ailleurs, pour autant qu'on puisse en juger dans / (p. 47) une œuvre au style stéréotypé, maîtrise la règle du réfléchi ${ }^{4}$, paraît entériner une dualité : "le mot oiseau s'explique par le comportement des oiseaux réels". Mais, s'il ne s'agit pas d'une bévue syntaxique isolée, il n'y a aucune théorisation derrière cette pratique de toute façon sporadique.

Il faut attendre la fin de l'Antiquité et l'un de ses plus grands génies, Augustin, pour trouver les premières questions logiques relatives au statut de l'autonyme. Dépassant les catégories de la dialectique stoïcienne et de l'Organon aristotélicien ${ }^{5}$, allant plus loin que la simple distinction signifiant/signifié, l'évêque d'Hippone, dans son traité à peine ébauché $D e$ dialectica (387) et dans son dialogue De magistro (389), pose des jalons fondamentaux pour la constitution d'une linguistique et d'une sémiotique du signe.

1.1 quatre niveaux d'analyse du signe : la quadripartition augustinienne

Pour la première fois en latin sont établies des distinctions entre mot ordinaire, mot métalinguistique en usage, mot autonyme et mot métalinguistique autonyme. Les mots ordinaires forment tout le lexique des énoncés en usage ; les mots métalinguistiques sont les termes servant à parler du langage ; les mots autonymes sont les mots de la première série dans des énoncés en mention; enfin les mots métalinguistiques autonymes sont les mots de la deuxième série dans des énoncés en mention ${ }^{6}$. À la terminologie près, on a chez lui toutes ces nuances subtiles.

Augustin définit ainsi quatre types de relation au mot :

Cum ergo uerbum ore procedit, si propter se procedit id est ut de ipso uerbo aliquid quaeratur aut disputetur, res est utique disputationi quaestionique subiecta, sed ipsa res /uerbum/ uocatur. Quidquid autem ex uerbo non aures sed animus sentit et ipso animo tenetur inclusum, /dicibile/ uocatur. Cum uero uerbum procedit non propter se sed propter aliud aliquid significandum, /dictio/ uocatur. Res autem ipsa, quae iam uerbum non est neque uerbi in mente conceptio, siue habeat uerbum quo significari possit, siue non habeat, nihil aliud quam /res/ uocatur proprio iam nomine. Haec ergo quattuor distincta teneantur, uerbum dicibile dictio res (Aug. Dialec. V).

"Lorsque, donc, un mot est prononcé, s'il est prononcé pour lui-même de façon à ce qu'il y ait question ou débat sur le mot lui-même, alors de toute façon c'est une chose qui est soumise à débat et à question ; mais cette chose a précisément le nom de /verbum/. Quant à ce

Peut-être faut-il comprendre un rapport phonique direct entre $a$-uis et $a(b)+$ uenio ; peut-être, implicitement, la source phonique est-elle l'expression a uia, "en s'éloignant du chemin"?

cf. FEST. 113, 25 : Magnum socerum appellat uir uxoris suae auum vs 112, 27 : Materfamiliae non ante dicebatur quam uir eius paterfamiliae dictus esset; 135,13 vs 135, $27 ; 139$, 2, etc.

Cf. Baratin 1989 a.

Nous suivrons, y compris pour les textes latins, l'usage typographique de Rey-Debove 1997: les autonymes seront désormais encadrés de barres. Ainsi comme chez elle, p. 29, "on appellera 'mots métalinguistiques' (stricto sensu), les mots du type adjectif, illisible, dire, et 'mots autonymes', les mots du type /table/; et on réservera l'expression 'mots métalinguistiques autonymes' aux mots du type /adjectif/, /illisible/, /diref'. Rappelons que, selon le même auteur (ibid.), "le métalexique d'une langue comprend donc des mots métalinguistiques et des mots autonymes, homonymes de tous les autres". 
qui est perçu, dans le mot (verbum), non par les oreilles / (p. 48) mais par l'esprit, et qui est contenu dans l'esprit même, il reçoit le nom de /dicibile/. En revanche, quand le mot est prononcé non pas pour lui-même mais pour signifier autre chose, il reçoit le nom de /dictio/. Quant à la chose même, qui n'est pas le mot ni le concept mental du mot, qu'elle ait un mot susceptible de la désigner ou non, elle ne peut recevoir que le nom de /res/, ici dans un emploi précis. On a donc ces quatre notions distinctes : verbum, dicibile, dictio, res" 7 .

Suivent les définitions de ces quatre notions :

Quod dixi /uerbum/ et uerbum est et /uerbum/ significat. Quod dixi /dicibile/ uerbum est, nec tamen /uerbum/, sed quod in uerbo intellegitur et animo continetur, significat. Quod dixi /dictionem/ uerbum est, sed quod iam illa duo simul id est et ipsum uerbum et quod fit in animo per uerbum significat. Quod dixi /rem/, uerbum est, quod praeter illa tria quae dicta sunt quidquid restat significat.

"Le terme /verbum/ ('mot') est un mot et signifie 'mot'. Le terme /dicibile/ est un mot mais ne signifie pas 'mot'; il signifie ce qui, dans le mot, est perçu et contenu dans l'esprit. Le terme /dictio/ est un mot mais qui signifie en même temps les deux précédents, à savoir le mot (verbum) et ce qui se produit dans l'esprit du fait du mot. Quant au terme /res/, c'est un mot qui signifie tout le reste hormis les trois premiers termes".

Baratin 1989 b (220) commente ainsi ce passage :

"L'analyse de l'expression élémentaire, intitulée de loquendo ('domaine de la dénomination'), est introduite par un examen de la nature même du mot, défini comme étant un signe. Cette définition, en intégrant le mot à une relation de signification, conduit Augustin à dissocier les différents aspects de cette relation :

- uerbum : le mot 'en lui-même', c'est-à-dire la séquence phonique porteuse d'une signification, mais considérée indépendamment de cette relation de signification ;

- dicibile : ce qui est désigné par le mot ;

- dictio : le mot comme assemblage d'un signifiant et d'un signifié ;

- res : la 'chose', c'est-à-dire le référent".

La définition augustinienne de dictio, en effet, semble insister sur l'idée qu'il s'agit d'une combinaison : dictio $=$ verbum + dicibile. Or le dicibile, par l'activité cognitive qu'il décrit de reconstruction mentale du sens par décodage, paraît s'apparenter au signifié de la linguistique saussurienne. Il est du coup tentant d'associer le verbum au signifiant (encore que la définition même, citée dans les passages ci-dessus, n'en dise rien) et, partant, la dictio au signe saussurien : dictio ("signe") = verbum ("signifiant") + dicibile ("signifié"). Ce triptyque s'oppose à res, le référent.

Pourtant, l'assimilation de verbum au concept de signifiant me paraît insuffisante. / (p. 49)

D'abord, /verbum/ est un terme grevé de polysémie, y compris dans les traités de dialectique. Le mot, métalinguistique, désigne évidemment n'importe quelle espèce de mot. Il désigne aussi, dans un emploi métalinguistique spécifique (qu'on pourrait dire hyponymique), le verbe ${ }^{8}$. Il désigne, on l'a vu ci-dessus, l'un des quatre modes de désignation ("signifiant" ?). Il désigne enfin le mot métalinguistique autonyme $/$ verbum $/{ }^{9}$. Du

Traduction personnelle, qui entreprend de coller au texte. On peut évidemment préférer l'excellente et moderne traduction proposée par Baratin \& Desbordes (1981), p. 215 ; les quatre notions, traduites, y deviennent respectivement, mot, dicible, dit et chose.

8/(p. 49) Aug. Mag. 4, 9 : Dicimus et alio modo /verbum/, quo significantur ea quae per tempora declinantur, ut /scribo//scripsi/, /lego/ /legi/, quae manifestum est non esse nomina, "nous utilisons aussi le terme /verbum/ dans un autre sens, pour signifier la catégorie qui se conjugue en temps, comme /scribo/ /scripsi/, /lego//legi/, qui, clairement, ne sont pas des noms". significari "tu es donc d'accord pour dire que quand nous disons $/$ erbum/, c'est un nom qui est 
coup, pour éviter toute équivoque sur le niveau d'analyse du métalexique où il se situe, Augustin utilise fréquemment, au lieu de /verbum/, un tour concurrent Cum dicimus $/ X /$, avec $X$ autonyme :

Mag. 4, 7 : ...cum dicimus /Lapis/; hoc enim uerbum etc. [donc cum dicimus /Lapis/= uerbum /Lapis/] ;

Mag. 4, 10: Scisne etiam, cum dicimus, /Animal/, aliud esse hoc trisyllabum nomen, quod uoce prolatum est, aliud id quod significatur? "sais-tu aussi que, lorsque nous disons /animal/, le nom trisyllabique qui est proféré est une chose et ce qui est signifié une autre chose ?" [on rejoint ici l'opposition verbum / dicibile ; cela revient à dire "le verbum /animal/ est autre chose que le dicibile /animal/'; donc cum dicimus /animal/ est une variante de verbum /animal/];

Ibid. un peu plus bas : nam cum dicimus $/$ signum/... ; etc. ${ }^{10}$

Or dans cette situation, on observe que le tour cum dicimus $/ X /$, équivalent de verbum dans son emploi technique, est souvent accompagné de descriptions phonologiques qui paraissent exhiber du signe mentionné principalement sa dimension signifiante : on l'a vu dans un exemple ci-dessus, où le verbum /animal/ est caractérisé comme un mot trisyllabique. On le voit souvent / (p. 50) ailleurs : hoc quadrisyllabum signum, cum dicimus /coniunctio/ (Mag. 4, 10), in hoc dissyllabo cum dicimus /Verbum/ (ibid.), iis duabus syllabis quas edimus cum dicimus /Verbum/ (4, 9), tres istas litteras quas enuntiamus cum dicimus /est/ (5, 14), etc.

Cette insistance sur la matérialité phonique ou graphique qui accompagne l'analyse qu'on doit faire du terme /verbum/ (via sa variante cum dicimus /X/) corrobore apparemment l'assimilation de verbum à signifiant.

Mais la chose n'est pas si simple. Verbum est fréquemment assimilable, chez Augustin, au concept d'autonyme : est verbum ce qui est proféré en soi et pour soi (propter se, dans Dialec. V), ce qui est signe de soi-même (seipsum significare : Mag. 5, 10 [bis] ; 6, 17 ; 6, 18 $[$ ter $]$ ).

\section{2 système des signes}

Augustin, en effet, remarque finement que les mots sont des signes; mais tous les signes ne sont pas des mots. Dans le système des signes (gestes, dessins, mots, etc.), les mots forment un système spécial, celui des signes langagiers. Parmi eux, certains désignent des signes non langagiers (par exemple le mot gestus "geste" ${ }^{11}$ ), d'autres désignent des signes langagiers (ce sont les mots métalinguistiques), les plus nombreux désignent le monde extralinguistique :

signifié" ; Mag. 5, 13 : Si autem a me quaeras quae sit pars orationis, Nerba/, nihil aliud respondebo quam, Nomen, "si en revanche tu me demandais quelle partie du discours est /Verba/, ma réponse serait nécessairement "un nom", ; etc.

Cette lourdeur, au demeurant, peut avoir un avantage de clarté ; car comme il est souvent question de verbum, faute d'une typographie adaptée en latin, il est indispensable de distinguer les emplois métalinguistiques (verbum) des emplois métalinguistiques autonymes (/verbum/). Voyons la manière de procéder dans un contexte court: Mag. 5, 11 : Nerbal enim cum dicimus, omne quod articulata uoce cum aliquo significatu profertur, significamus; unde omne nomen, et ipsum cum dicimus : /nomen/, uerbum est : at non omne uerbum nomen est, quamuis nomen sit, cum dicimus : /uerbum/ "Quand nous disons /verba/, nous signifions tout ce qui est proféré par la voix articulée avec une quelconque signification. C'est pourquoi tout nom (nomen), y compris quand nous disons /nomen/, est un verbum, mais tout verbum n'est pas un nom, même si quand nous disons /verbum/ c'est un nom". L'usage (hérité de Rey-Debove 1997) des barres obliques pour matérialiser l'autonyme montre que, dans ce court paragraphe, l'autonyme /verbum/ et l'autonyme /nomen/ sont systématiquement accompagnés de cum dicimus, alors que l'emploi en usage des mêmes termes métalinguistiques est caractérisé par l'absence de cette indication. Cum dicimus : /uerbum/ revient à écrire uerbum /uerbum/.

\footnotetext{
11 / (p. 50) Ce sont les mots métasémiotiques : Rey-Debove 1997 (37).
} 
Videtur ergo mihi loquendo nos aut uerba ipsa signare uerbis, aut alia signa, uelut Igestum/ cum dicimus aut /litteram/; nam his duobus uerbis quae significantur, nihilominus signa sunt : aut aliquid aliud quod signum non sit, uelut cum dicimus, /Lapis/; hoc enim uerbum signum est, nam significat aliquid, sed id quod eo significatur, non continuo signum est (Mag. 4, 7).

"Il me semble donc qu'en parlant, nous signifions par les mots, ou bien les mots euxmêmes, ou bien d'autres signes, comme lorsque nous parlons de gestes ou de lettres car les choses signifiées par ces deux mots n'en sont pas moins des signes : ou bien nous signifions autre chose qui n'est pas un signe, comme en disant pierre : ce mot en effet est un signe, car il désigne un objet, mais cet objet qu'il signifie n'est pas pour autant un signe" (traduction prise dans Rey-Debove 1987 (37)).

Du simple fait de la référence, puisqu'ils désignent automatiquement leur référent, les mots (comme les gestes) sont des signes ; certains d'entre eux sont des "signes de signes" (l'expression signorum signa est dans Augustin, Mag. 4, 8; signi signum en 4, 9) : ils constituent tout le métalexique. Dans ce métalexique figurent à la fois les mots métalinguistiques (nomen, uerbum, coniunctio, etc.) et métasémiotiques (gestus), mais aussi les autonymes.

Pour cette catégorie si particulière de l'autonyme, Augustin ne mène pas l'analyse à son terme; il remarque que certains mots métalinguistiques ont un statut particulièrement inclusif : signum signifie "signe" et est un signe; idem pour verbum (qui est un mot) ou nomen (qui est un nom) ; au contraire, coniunctio est un nomen, un verbum, un signum, mais non pas une conjonction : / (p. 51)

Mag. 4, 10: Ad. - (...) nam cum dicimus, /Signum/, non solum signa caetera quaecumque sunt, sed etiam seipsum significat; est enim uerbum, et utique omnia uerba signa sunt.

Aug. - Quid? in hoc disyllabo cum dicimus, /Verbum/, nonne tale aliquid contingit? Nam si omne quod cum aliquo significatu articulata uoce profertur, hoc disyllabo significatur, etiam ipsum hoc genere includitur.

Ad. - Ita est.

Aug. - Quid? /nomen/ nonne similiter habet? Nam et omnium generum nomina significat, et ipsum nomen generis neutri nomen est. An, si ex te quaererem quae pars orationis /nomen/, posses mihi respondere recte, nisi,/nomen/?

Ad. - Verum dicis.

Aug. - Sunt ergo signa quae inter alia quae significant, et seipsa significent.

Ad. - Sunt.

Aug. - Num tale tibi uidetur hoc quadrisyllabum signum, cum dicimus : /coniunctio/?

Ad. - Nullo modo : nam ea quae significat, non sunt nomina; hoc autem nomen est.

"Adéodat: - (...) car, quand nous disons /Signe/, ce ne sont pas seulement tous les signes qui existent que nous signifions, mais aussi lui-même; car c'est un mot et de toute façon tous les mots sont des signes.

Augustin : - Quoi ? et pour le dissyllabe /verbum/ n'est-ce pas la même chose ? car si tout ce qui est proféré en association avec un sens par la voix articulée est signifié par ce dissyllabe, alors lui aussi est inclus dans ce genre.

Adéodat : - C'est vrai.

Augustin : - Quoi ? n'est-ce pas le même comportement pour /nomen/? Car il signifie les noms de tous genres et lui-même est un nom de genre neutre. Si je te demandais la nature de /nomen/, pourrais-tu répondre autre chose que "c'est un nom"?

Adéodat : - Tu dis vrai.

Augustin: - Il y a donc des signes qui, au milieu des signes qu'ils signifient, se signifient également eux-mêmes. 
Adéodat : - Il y en a.

Augustin : - Est-ce la même chose, d'après toi, quand nous disons /coniunctio/?

Adéodat : -Non, pas du tout : car ce que signifie /coniunctio/ ce ne sont pas des noms, mais lui-même est un nom" ${ }^{12}$. / (p. 52)

On voit que la dialectique augustinienne est très proche d'une solution mais qu'il lui manque encore des distinctions de niveau d'analyse. C'est ce que remarque Rey-Debove 1997 (37) :

"Cette possibilité est le fait de la contingence linguistique, et la plupart du temps il n'y a aucune coïncidence : morphème est un mot, et non un morphème, adverbe est un nom et pas un adverbe, etc. D'autre part, l'interprétation d'Augustin est discutable car si un mot se signifie lui-même, il signifie son expression et son contenu. Signe ne se signifie pas lui-même, car dans ce cas le seul signe existant serait signe. Il signifie un signe dont l'expression et le contenu sont indéterminés, c'est-à-dire un multisigne. Signe et /signe/ sont dans un rapport d'inclusion, mais non de signification. Le signe peut être signe comme le siège peut être un fauteuil. On dira donc que le signe signe se classe lui-même dans sa classe, que signe est un hyperonyme de /signel'.

Faute de bien faire la part entre signe de signes, multisigne, signe de multisigne (cf. l'extraordinaire sophistication du tableau de Rey-Debove 1997 (44), dans lequel /autonyme/, dans un niveau 5 de langage ou métalangage de niveau 4, est présenté comme un signe de signe de multisigne de signe...), Augustin, comme beaucoup de nos contemporains, reste dans une équivoque relative. Il lui manque une formulation comparable à celle de Rey-Debove 1997 (passim) selon laquelle l'autonyme se signifie tel qu'il est à l'étage inférieur de métalangage ${ }^{13}$. En disant Id est quod dictum est nomen seipsum significare cum aliis quae significat "Voilà ce que nous voulons dire en disant que le nom se désigne lui-même en même temps qu'il désigne d'autres choses que lui-même" (Mag. 6, 18), Augustin parle en même temps de nomen et de /nomen/. Il veut dire que l'autonyme de mot métalinguistique /nomen/ est un nom et que le mot métalinguistique nomen est le terme générique qui sert d'étiquette à tous / (p. 53) les noms. Mais sa formulation est l'objet d'un amalgame sémiotique ${ }^{14}$ : la même occurrence de nomen est à interpréter à la fois comme un nom métalinguistique en usage et comme un autonyme ${ }^{15}$.

Cf. aussi Mag. 6, 18 : /Coniunctio/ (...) nomen inter illa quae significat numerari non potest. (...) "Le nom /conjonction/ ne peut pas être rangé parmi les choses qu'il désigne". / (p. 52)

C'est-à-dire que "le signe se signifie tel qu'il serait s'il ne se signifiait pas" (Rey-Debove 1997, p. 297). Augustin, dans le De dialectica X, sent pertinemment que le mot autonyme est homonyme du mot en usage et semble établir une hiérarchie métalinguistique. Comparant différents "sens" métalinguistiques de Tullius, il dit que c'est 1 . le nom d'un orateur célèbre, 2 . un pied dactylique, 3 . un verbum dans lequel les sens précédents et lui-même sont en position d'homonymie (aequiuoca) : Possum enim recte dicere: /Tullius/ est nomen, quo significatur homo, summus quidam orator (...). Item responderi possem hoc modo: /Tullius/ est pes dactylus his litteris constans - quid enim nunc opus est litteras enumerare? Licet etiam dicere : /Tullius/ est uerbum, per quod aequiuoca inter se sunt omnia cum hoc ipso quae supra dicta sunt et si quid aliud inueniri potest. Baratin \& Desbordes 1981 (227) traduisent ainsi la dernière phrase : "Tullius est un mot où toutes les significations que je viens d'énumérer (et d'autres éventuellement) se retrouvent en tant qu'homonymes, entre elles et avec ce terme lui-même". On voit le sens particulier de verbum : c'est le mot dans sa complétude sémantique, qui subsume tous les sens réalisables du mot en discours. Quelque chose qui peut s'apparenter à l'entrée de dictionnaire des lexicographes modernes. Mais en disant que cet élément, qui appartient à une strate supérieure de métalangage, est lui-même homonyme de ses occurrences de strates inférieures, Augustin a une formulation proche de Rey-Debove 1997 (29), selon laquelle les "mots autonymes [sont] homonymes de tous les autres". / (p. 53)

Terminologie de Rey-Debove 1997 (276) ; exemples de ce type de cumul dans Nicolas 2000 (56-57).

Cette méprise, habituelle chez les modernes, est à peu près constante chez les Antiques. Citant une définition tirée du l'anonyme Tractatus De Categoriis Aristotelis, dans l'Aristoteles Latinus, I, 1-5, 
C'est la raison pour laquelle son personnage peut dire d'une manière toute sophistique à Adéodat que tout nomen est un verbum et que tout verbum est un nomen : en 4, 9 il est conclu par Augustin que tout nomen est un verbum ${ }^{16}$ (c'est-à-dire que tout nom est un mot) : verbum dans son sens général de "mot" (son sens spécifique de "verbe" n'étant ici pas concerné) est un mot métalinguistique plus générique que nomen, car on peut qualifier de verbum ou verba n'importe quel mot ou énoncé ${ }^{17}$. Mais en 5,11 il prouve au jeune élève que tout verbum est un nomen, ce qui contredit le système d'inclusion lexicale reconnu quelques paragraphes plus haut : c'est qu'alors il faut comprendre qu'il évoque l'autonyme $/$ erbum/ ${ }^{18}$. / (p. 54)

\section{3. statut logique et nature grammaticale de l'autonyme}

Malgré quelques insuffisances aux yeux des plus pointus des spécialistes contemporains, la dialectique d'Augustin frappe par son modernisme et sa lucidité. On y trouve notamment deux acquis remarquables : 1) le même élément langagier change de statut selon l'énoncé (c'est-à-dire selon le niveau de [méta]langage) dans lequel il s'insère ; 2) les termes cités pour eux-mêmes (donc nos autonymes, auxquels il ne parvient pas à faire coller un terme univoque approprié, et qu'il appelle souvent uerba) sont grammaticalement des noms.

\subsection{1. variabilité d'un même élément selon l'énoncé}

Pour le premier point, il l'illustre aussitôt après avoir donné les définitions des quatre modes de désignation rappelés ci-dessus, verbum, dicibile, dictio et res :

Dialec. V: Sed exemplis haec inlustranda esse perspicio. Fac igitur a quoquam grammatico puerum interrogatum hoc modo: '/arma/ quae pars orationis est?' Quod dictum est /armal, propter se dictum est, id est uerbum propter ipsum uerbum. Cetera uero, quod ait 'quae pars orationis', non propter se, sed propter uerbum, quod /arma/ dictum est, uel animo

135 : Omne nomen, licet proprium, quod possit esse commune cum ceteris, homonymon uocari; ut Cicero, non unus sed plures, "tout nom, même propre, qui peut être commun à plusieurs <êtres>, est appelé homonyme ; par exemple Cicéron, qui n'est pas un mais plusieurs”, Desbordes 1988 (62) écrit : "Une définition peu soignée, comme celle-ci, ne se soucie pas de marquer une différence entre mot 'en mention' et mot 'en usage', en profitant du fait qu'un mot sur lequel on attire l'attention du point de vue métalinguistique, peut continuer à renvoyer à autre chose qu'à lui-même : on comprend que Cicéron peut être dit non d'un seul individu Cicéron, mais de plusieurs Cicéron(s). Ailleurs cette négligence peut avoir des conséquences plus graves, outre qu'elle pose des problèmes insolubles au traducteur". Augustin $($ Mag. 8,23) cite à cet égard le bon mot d'un orateur : celui-ci fait reconnaître à son adversaire que c'est de notre bouche que sort toute parole articulée ; or l'adversaire a prononcé /lion/; donc c'est un lion qui est sorti de sa bouche... Le jeune Adéodat s'insurge à juste titre : il s'agit là, dit-il, d'une confusion entre mot et chose.

Ergo et nomen uerbum est.

Mag. 4, 8 : Cum dicimus disertum hominem bonis uerbis uti, etiam nominibus utique utitur; et cum seni domino apud Terentium seruus retulit: Bona uerba quaeso, multa ille etiam nomina dixerat, "quand nous disons qu'un homme éloquent utilise les mots justes, il utilise aussi des noms ; quand chez Térence l'esclave dit à son vieux maître Bona uerba quaeso, l'autre avait dit de nombreux noms". Donc verbum subsume nomen.

Mag. 5, 11 : Tu ergo nescis, cum dicimus /nomen/ et /uerbum/ duo uerba nos dicere? Ad. : - Scio. Aug. : - Quid? illud nescis, cum dicimus /nomen/ et /uerbum/ duo nomina nos dicere? Ad. : - Id quoque scio. Aug. : - Scis igitur tam nomen uerbo quam etiam uerbum nomine significari, "Tu ignores donc que quand nous disons /nomen/ et /verbum/, nous disons deux mots ? Ad. : - Non, je le sais. Aug. : - Et alors ? ignores-tu que quand nous disons /nomen/ et /verbum/, nous disons deux noms ? Ad. : - Non, je le sais aussi. Aug. : - Alors tu sais que le nom est autant signifié par le mot que le mot par le nom". C'est un syllogisme : ce sont les autonymes /nomen/ et /verbum/, et eux seuls, qui peuvent être dits indifféremment "mots" ou "noms". Adéodat, un peu aidé par le maître, ne s'y trompe pas, qui signale, ibid., que tout nom (y compris l'autonyme /nomen/) est un mot mais que tout mot n'est pas un nom (seul l'autonyme /verbum/ est un nom). / (p. 54) 
sensa uel uoce prolata sunt. Sed cum animo sensa sunt, ante uocem dicibilia erunt; cum autem propter id quod dixi proruperunt in uocem, dictiones factae sunt. Ipsum uero /arma/ quod hic uerbum est, cum a Vergilio pronuntiatum est, dictio fuit; non enim propter se prolatum est, sed ut eo significarentur uel bella quae gessit Aeneas uel scutum et cetera arma quae Vulcanus heroi fabricatus est. Ipsa uero bella uel arma, quae gesta aut ingestata sunt ab Aenea - ipsa inquam quae cum gererentur atque essent uidebantur quaeque si nunc adessent uel digito monstrare possemus aut tangere, quae etiamsi non cogitentur non eo tamen fit ut non fuerint - ipsa ergo per se nec uerba sunt nec dicibilia nec dictiones, sed res quae iam proprio nomine /res/ uocantur.

"Mais je vois qu'il faut illustrer ces propos par des exemples. Imagine qu' un professeur interroge un élève ainsi : '/arma/ c'est quelle nature de mot ?' ; ce qu'on dit en disant /arma/ est dit en soi et pour soi, c'est un mot (verbum) en fonction du mot même, alors que le reste de sa phrase 'c'est quelle nature de mot ?' n'est pas conçu par l'esprit ni prononcé par la voix en soi et pour soi mais en fonction du mot (verbum) /arma/. Mais lorsqu'ils sont conçus par l'esprit et non encore proférés par la voix, ils sont des dicibilia. Et, pour la raison susdite, quand ils ont surgi sous forme vocale, ils sont devenus des dictiones. Arma lui-même, qui ici est un verbum, quand il a été prononcé par Virgile, était une dictio car il n'a pas été proféré en soi et pour soi mais pour signifier les guerres menées par Énée ou le bouclier et toute la panoplie que Vulcain a réalisés pour le héros. <Enfin> ces guerres mêmes et ces armes qui ont été portées ou menées par Énée - je veux dire celles qui, lorsqu'elles étaient portées et qu'elles existaient, étaient visibles, et, si elles étaient là, on pourrait les montrer du doigt et, même si on n'arrivait pas à se les représenter, elles n'en seraient pas moins réelles -, ces choses mêmes donc, en elles-mêmes, ne sont ni des verba, ni des dicibilia, ni des dictiones mais bien des choses qui sont appelées spécifiquement /res/" 19 . / (p. 55)

On voit donc que, selon l'énoncé où il s'insère et la réalité qu'il désigne, l'élément arma acquiert un statut différent.

1.3.2. caractère nominal de l'autonyme

Le deuxième acquis important de la sémiotique augustinienne, au moins pour ce qui concerne la question de l'autonymie, est la preuve grammaticale administrée par le maître que les autonymes sont tous des noms : quelle que soit la nature de la dictio concernée, elle est un nomen dès qu'elle est utilisée en mention (donc dès qu'elle est utilisée comme un verbum, dans l'un de ses sens probables). Pour en convaincre son jeune auditeur, le personnage d'Augustin du De magistro demande à Adéodat de se livrer à de l'analyse logique :

Mag. 5, 15 : "Quia homo est, animal est" ; nonne temere dicerem?

Ad. - Temere omnino : sed non temere plane diceres : "Si homo est, animal est".

Aug. - Recte dicis. Itaque in locutione tua placet mihi /Si/ ; placet et tibi : utrique autem nostrum in mea displicet /Quia/.

Ad. - Assentior.

Aug. - Vide iam utrum istae duae sententiae plena pronuntiata sint: "Placet /Si/", “Displicet /quial”.

Ad. - Plena omnino.

Aug. - Age, nunc dic mihi quae ibi sint uerba, quae nomina.

Ad. - Verba ibi uideo esse /placet/, et /displicet/: nomina uero quid aliud quam /si/, et lquial?

Aug. - Has ergo duas coniunctiones etiam nomina esse satis probatum est.

"N'aurais-je pas quelque témérité à dire : 'parce que c'est un homme, c'est un animal' ? 
Adéodat : - Si, tout à fait ; mais tu pourrais dire sans aucune témérité : 'Si c'est un homme, c'est un animal'.

Augustin : - Tu as raison. Aussi dans ton énoncé, je trouve que /si/ convient, et toi aussi; en revanche, tous les deux, dans mon énoncé, nous trouvons que /parce que/ ne convient pas.

Adéodat : - Je suis d'accord.

Augustin: Examine maintenant si ces deux phrases sont bien constituées: '/Si/ convient', '/Parce que/ ne convient pas'.

Adéodat : - Elles le sont.

Augustin : - Alors dis-moi où, dans ces phrases, se trouvent les verbes et les noms.

Adéodat : - Comme verbes je vois /convient/ et /ne convient pas/. Comme noms, il n'y a que /Si/ et /Parce que/.

Augustin: - Donc ces deux conjonctions sont aussi des noms, la preuve en est faite". / (p. 56)

Et plus loin, en bonne méthode, il démontre que ce qui est vrai pour des conjonctions l'est pour toutes les parties du discours, qui peuvent se retrouver en position de noms dès qu'elles sont en mention. En effet, on peut utiliser le verbe nomino (qui inclut nomen) à côté de pronoms, adverbes, etc. Il suffit de poser un énoncé correct comme "Ce que nous appelons $X$, les Grecs l'appellent $Y$ " et de mettre comme variable pour $X$ et $Y$ un pronom (Quis et tís), un verbe (volo et boúlomai), un adverbe (bene et kalôs), un participe (scriptum et gegramménon), une conjonction (et et kai), une préposition ( $a b$ et apò), une interjection (heu et $o i)$. Et voilà qu'en autonymie, tous ces éléments non nominaux fonctionnent comme des noms $\left(\right.$ Mag. 5, 15) ${ }^{20}$.

1.3.3. lacune dans le système : la question du cas, du genre et du nombre

Cette réflexion d'Augustin est absolument capitale. Mais elle ne va pas plus en avant sur la question des cas, genre et nombre. En effet, si quia ou et sont susceptibles d'occuper une fonction de substantifs, s'ils sont des noms occasionnels, ils doivent avoir un genre et un nombre sur lesquels induire tous les phénomènes d'accord et doivent pouvoir être virtuellement casualisés.

Spontanément, il paraît aller de soi que ces éléments non nominaux, quand ils occupent la place d'un nom, doivent engager des accords au neutre singulier : par essence, ils ne sont ni masculins ni féminins, ni l'un ni l'autre (neutrum). C'est ce que l'on observe universellement chez les auteurs latins : ces éléments sont ainsi volontiers déterminés par hoc ou illud ${ }^{21}$ et les accords d'éventuels participes sont systématiquement calculés au neutre. Mais Augustin ne le dit pas.

La seule indication de genre qui soit donnée à cet égard dans le De magistro concerne l'autonyme nomen (Mag. 4, 10), dans un passage où il remarque la spécificité logique des signes de signes : Quid? Inomen/ nonne similiter habet? Nam et omnium generum nomina significat, et ipsum nomen generis neutri nomen est, "Quoi? n'est-ce pas le même comportement pour /nomen/? Car il signifie les noms de tous genres et lui-même est un nom de genre neutre". Nomen, en tant qu'étiquette terminologique, subsume tous les noms du lexique, y compris les féminins et les masculins, bien que lui-même soit un mot de genre

Et même, en complétant le raisonnement, un adverbe grec, dans un énoncé tel que celui qu'Augustin utilise, fonctionne comme un nom à l'intérieur d'un énoncé latin, donc comme un nom latin : si l'on dit "pour volo les Grecs disent boúlomai", notre phrase est un énoncé français dans laquelle deux verbes appartenant à des langues étrangères sont respectivement le régime d'une préposition et l'objet du verbe : ce sont donc, en bonne logique, des noms français (dans ce contexte). 
neutre. Mais on voit bien qu'ici ce n'est pas la neutralité de l'autonyme qui est en cause : nomen est un nom neutre, indépendamment de ses emplois autonymiques possibles.

On aimerait bien qu'Augustin nous dise clairement que tout autonyme, y compris un autonyme de nom commun, est de genre neutre dans l'énoncé où / (p. 57) il figure (comme en français - où le genre neutre est assumé par le masculin - lorsque l'on dit : "Femmes est féminin pluriel"). Mais même un auteur capable d'analyses aussi performantes que l'évêque d'Hippone s'arrête en chemin avant de poser cet axiome qui nous paraît si naturel.

La raison se trouve à coup sûr dans la typologie de la langue latine même : le nom stricto sensu a un genre, un nombre et un cas et il paraît extrêmement difficile à un latinophone de dépasser ce dogme grammatical. S'il faut mentionner un nom, que, ce faisant, on transforme en autonyme, on a du mal à faire abstraction de ses genre et nombre intrinsèques, c'est-à-dire des genre et nombre du mot en usage. De même avec le cas : autonyme ou non, c'est un substantif qui occupe une place dans l'énoncé et son cas est très généralement déterminé par la syntaxe du langage ordinaire.

Ainsi, sur la question du cas, Augustin lui-même hésite entre une démarcation franche de l'élément autonyme sur le plan syntaxique et une absence de démarcation. Il peut ou bien laisser l'autonyme au nominatif (ici senti comme le degré zéro de la casualité ${ }^{22}$ ), dans une apesanteur syntaxique :

Mag. 3, 5 : ...cum dicimus /paries/...; 4, 7 : ...cum dicimus /Lapis/.. ; 4, 10 : ...cum dicimus /coniunctio/...; 5, 13 : ...cum dicimus /hic uir/, /ipse rex/, leadem mulier/, /hoc aurum/, /illud argentum/...; etc.

ou bien l'accorder au cas où l'appelle son rôle dans la phrase (souvent l'accusatif) :

Mag. 4, 7 : .../gestum/ cum dicimus uel /litteram/... "quand nous disons /gestus/ ou /litteraf"; 10, 33: ...cum primo audirem legeremue /sarabaras/, "la première fois que j'entendais ou lisais le mot/sarabaraef' ${ }^{23}$.

Cela l'amène, éventuellement, dans un comique involontaire, à entériner jusque dans son commentaire la variabilité morphologique de l'autonyme, comme dans ce passage où l'autonyme /homo/ change de cas, donc de nombre de syllabes :

Mag. 8, 24: si (...) quid esset homo requireret (...) ad id quod duabus syllabis significatur, animus curreret ; (...) quid enim metuam /hominem/, id est tres istas syllabas non esse me confiteri ? (...) ...cum dicitur, 'Non es igitur homo' (...) non possum non putare ad id conclusionem referri, quod his duabus syllabis significatur, "si on cherchait juste ce qu'est un homme (ou 'ce qu'est /hommel', avec ambiguïté volontaire ici), l'esprit irait <prioritairement> vers ce qui est signifié par ces deux syllabes (ici homo) ; (...) car pourquoi devrais-je craindre/(p. 58) d'avouer que je ne suis pas le mot homo, avec ses trois syllabes (ici hominem) ; (...) quand on dit 'tu n'es pas un homme' (ici homo), je ne peux pas m'empêcher de penser que la conclusion se rapporte à ce qui est signifié par ces deux syllabes..." 24 .

1.3.4. primauté du langage ordinaire sur le métalangage

Augustin se justifie ainsi de ces privautés à l'égard de la logique. Le langage étant fait de signes, la première réaction spontanée de l'allocutaire est d'activer mentalement, dans

C'est l'emploi que les grammaires décrivent comme le "nominatif dénominatif". Cf. notamment Serbat 1996 (32-45).

La citation à laquelle se réfère Augustin ici, tirée de Daniel 3, 94, et qui a été rappelée verbatim quelques lignes plus haut, comporte le nominatif sarabarae. Il s'agit, dans les références ci-dessus, d'autonymie lexicale (on cite un signe global) et non d'autonymie lemmatique (on cite une forme particulière du paradigme : sur cette typologie, cf. Nicolas 2003) : les accusatifs qu'on y lit n'ont d'autre raison que de répondre à la pression des verbes transitifs dont ces autonymes dépendent. / (p. 58)

Baratin \& Desbordes sont obligés, dans leur traduction, d'esquiver ce problème insoluble : ils ne tentent pas de traduire littéralement et, pour chaque occurrence ici signalée en gras, écrivent seulement "mot". 
l'opération de décodage, le seul signifié ${ }^{25}$. Il existe une règle naturelle prévalente qui fait que, dans la communication langagière, l'attention se porte sur le signifié, non sur le signifiant: Mag. 8, 24 : ... ea scilicet regula, quae naturaliter plurimum ualet, ut auditis signis ad res significatas feratur intentio, "en vertu de la règle, qui normalement l'emporte, et qui fait que quand on entend un signe, l'attention se porte sur ce qu'il signifie" ${ }^{26}$. Le langage ordinaire est le code par défaut, le métalangage est le code marqué. Le langage ordinaire prévaut sur le métalangage : si la question est quid est homo ?, il est naturel de comprendre qu'elle porte sur l'extra-linguistique ("qu'est-ce que l'homme ?") ; en revanche, si elle se formule quae pars orationis est homo?, elle insiste sur le statut de signe de l'autonyme /homo/ ("quelle est la nature du mot /homo/?") ${ }^{27}$. Le métalangage se doit donc d'être suffisamment contextualisé dans chaque énoncé. Par conséquent, dès que la clarté statutaire de l'énoncé est acquise, il n'y a pas nécessairement lieu d'insister sur le statut spécial de l'autonyme en le dotant de cette / (p. 59) apesanteur syntaxique remarquée plus haut : comme l'accord secondaire du segment autonymique, impliquant sa variabilité morphologique, ne nuit pas à la clarté de l'argument énoncé, on a sans doute intérêt à priviliégier une correction grammaticale apparente.

\section{Les deux manières de pratiquer le métalangage en latin}

Garantis par cette loi de linguistique générale qu'Augustin a exprimée dans les termes rappelés ci-dessus et que chaque latinophone applique spontanément sans l'exprimer, les auteurs latins ont deux manières de traiter avec le métalangage: l'une, que j'ai appelée "tendance métalinguistique", l'autre que j'ai nommée "tendance intégrationniste" 28 en empruntant l'expression à Guy Serbat ${ }^{29}$.

Un adepte de la tendance métalinguistique n'hésite pas à neutraliser le cas du terme en mention : dès lors qu'il est en mention, sa forme propre est auto-justifiée et sa fonction grammaticale dans l'énoncé est manifestée uniquement par le contexte :

PRISC. GL 2, 19, 5 : ...ut si dicam /Cremes/ pro /Chremes/, "comme quand je dis Cremes au lieu de Chremes..." (où le nominatif étiquette fait fonction respectivement d'accusatif et d'ablatif).

Le caractère non casualisable de l'élément mentionné ne constitue pas non plus un embarras, comme cela se voit de l'exemple suivant de Suétone dans lequel un premier infinitif

C'est ce que Recanati (1979) nomme la "transparence" du signe linguistique : sitôt proféré, le signifiant s'efface au profit de ce qu'il représente en terme de signifié ; et c'est grâce à cette transparence de l'étoffe signifiante que l'énoncé fait sens.

Traduction de Baratin \& Desbordes 1987 (236). De même, quelques lignes plus haut, Augustin évoque la règle conventionnelle selon laquelle l'esprit court vers la chose signifiée, placita illa loquendi regula ad id quod (...) significatur, animus curreret.

Cf. Mag. 8, 24 : si quaererem quid esset homo, responderes fortasse 'animal' ; si autem quaererem quae pars orationis esset homo, nullo modo posses recte respondere nisi, 'Nomen' : quamobrem cum homo et nomen, et animal esse inueniatur, illud dicitur ex ea parte qua signum est, hoc ex parte rei quam significat. Qui ergo quaerit utrum homo nomen sit, nihil ei aliud quam esse respondeam: satis enim significat ex ea parte se uelle audire, qua signum est, "si ma question portait sur ce qu'est un homme, tu répondrais peut-être 'un animal'; si elle portait sur la partie du discours de /homme/, tu ne pourrais faire autrement que de répondre 'un nom'; aussi puisque /homme/ se trouve être à la fois un nom et un animal, c'est que dans le premier cas la réponse porte sur cette partie qui fait que /homme/ est un signe, dans le deuxième cas sur cette partie qu'est la chose signifiée. Donc si on me demande si /homme/ est un nom, je dois répondre que oui, car il est assez clair que le locuteur souhaite évoquer la partie <du mot> par laquelle il est un signe”. Dans ce passage, signum représente le signe devenu opaque (Recanati 1979), le signe sur lequel l'esprit s'arrête de façon métalinguistique. C'est, comme verbum dans certains emplois, un équivalent presque complet du concept d'autonyme. / (p. 59)

Cf. Nicolas 2000 (48) et 2001 (500).

Cf. Serbat 1996 (34). / (p. 60) 
autonyme est un complément de type accusatif, alors qu'un deuxième est régime d'une préposition régissant l'ablatif et un troisième équivaut à un attribut au nominatif :

SuET. Aug. 87, 2 : Ponit adsidue (...) /betizarel pro /languerel, quod uulgo /lachanizarel dicitur "il met couramment /betizare/ à la place de /languere/, ce qui se dit communément /lachanizaref'...

La présence de pro suffit à donner à Cremes ou à languere une valeur ablative et une fonction cohérente dans l'énoncé, le "nominatif" lachanizare et l' "accusatif" betizare sont analysés comme tels dans le contexte. Et dans ces deux phrases, le contexte est nettement orienté vers le métalangage: Priscien écrit un ouvrage entièrement métalinguistique; Suétone, dans ce passage, évoque les habitudes langagières idiolectales d'Auguste. On n'est donc pas ici dans le code par défaut qu'est le langage ordinaire mais dans le code marqué qu'est le métalangage. On ne risque donc pas d'interpréter à solécisme ces contraventions manifestes à l'usage.

Mais un adepte de la tendance intégrationniste ne se contente pas de ces autonymes mal casualisés qu'il considère sans doute comme solécisants. À la translation logique fondamentale, qui consiste à transformer tout segment / (p. 60) autonymique en substantif, l'écrivain intégrationniste ajoute une translation supplémentaire qui consiste à faire de ce substantif, aussi souvent que possible, un nom accordé selon les règles usuelles. Ainsi, il corrigerait les énoncés métalinguistiques ci-dessus et écrirait sans doute respectivement $u t$ si dicam Cremetem pro Chremete et ...pro languendo...

On a vu qu'Augustin pratiquait les deux méthodes (...cum dicimus /Lapis/... vs .../gestum/ cum dicimus uel /litteram/...).

C'est la seconde méthode qui est très généralement adoptée par les Latins ${ }^{30}$ : l'autonyme, quand c'est possible, est très majoritairment casualisé selon les règles standard et gratifié des genre et nombre qui sont les siens dans le langage ordinaire. D'où ces innombrables énoncés métalinguistiques dans lesquels le caractère décalé de l'autonyme est gommé, où la mention se dilue dans l'usage. On peut dire, métaphoriquement, que la tendance métalinguistique s'apparente au discours direct (cum dicimus: 'Lapis'), la tendance intégrationniste au discours indirect (cum dicimus litteram) ${ }^{31}$.

En effet, quand on fait mention d'un élément quelconque de l'énoncé tel quel, on se met, analogiquement, dans la même situation que l'énonciateur qui cite en l'état les paroles d'autrui dans son propre discours. Le discours direct est l'émergence, dans l'énoncé en cours, de la voix d'un autre énonciateur (parfois soi-même, mais avec un décalage chronologique qui autorise à considérer qu'il s'agit bien d'un autre énonciateur) et cette polyphonie provoque une rupture syntaxique : ainsi la pause automatique qui sépare le verbe dicendi du discours direct tend à séparer le verbe de son complément d'objet, laquelle scission n'est pas réglementaire dans la langue. De la même façon, l'autonyme mentionné tel quel est précédé d'une pause, vérifiable dans les langues modernes et analogiquement extensible aux langues anciennes. La polyphonie, en l'espèce, tient à la présence dans l'énoncé normé d'un métalangage qui fonctionne peu ou prou comme une autre langue. Dans le cas du discours direct comme dans celui de l'"autonymie directe", le segment ainsi mis en relief par la pause et la rupture syntaxique vaut par sa matérialité signifiante : sont rapportées à l'identique dans l'énoncé en cours des paroles d'autrui, celles-ci et non pas d'autres, ou un terme en mention, celui-ci et non pas un de ses synonymes, d'une manière qui se revendique comme authentique. Cela peut provoquer de notables turbulences dans l'analyse logique de la phrase :

Non. 329, 5 : /Sexus/ masculini generis esse manifestum est "il est net que sexus est du genre masculin" (le sujet de l'infinitive est le nominatif autonyme sexus); 
Non. 276, 13 : /Victurus/ /uictoriam potiturus/ auctoritas prudentium putauit esse "l'autorité des spécialistes est d'avis que /victurus/vaut pour /victoriam potiturus/" (le sujet et l'attribut du sujet de la proposition infinitive se trouvent / (p. 61) être des lemmes autonymes au nominatif, expliquant un passage d'un écrivain de la République contenant la forme uicturus esset).

Le discours indirect, lui, supprime la pause et la rupture syntaxiques. La grammaire y reprend ses droits et le complément du verbe dicendi, qui prend l'allure d'une complétive, est intégré à l'énoncé principal : les règles de concordance des temps, les modifications d'embrayeurs et de tout ce qui concerne le hic et nunc concourent à unifier l'énonciation ; la transformation en récit (au sens benvenistien) des paroles d'autrui abolit la deuxième énonciation perceptible dans le discours direct et annule donc la polyphonie. De la même façon, l'autonyme traité à la manière intégrationniste perd peut-être sa pause à l'oral (il est difficile de le prouver) et à coup sûr les marques d'autonomie syntaxique, notamment d'imperméabilité au système casuel (si dicam /Cremes/ pro/Chremes/), qui matérialisent en creux son statut de mention. L'accord de l'autonyme (dicam /Cremetem/ pro /Chremete/) est analogiquement comparable aux modifications d'embrayeurs qui caractérisent le discours indirect et, comme lui, l'“autonymie indirecte” tend à gommer la polyphonie. Mais il n'y a théoriquement pas d'ambiguïté possible si le contexte général est suffisant : s'il est assez clair que l'on parle du langage, alors on saura bien retrouver la mention et la reconnaître malgré les accords secondaires qui la dissimulent, de la même façon qu'on est capable de reconnaître et de reconstituer du discours direct sous du discours indirect.

De là ces innombrables étymologies cicéroniennes grammaticalisées secondairement :

Tusc. 2, 43 : Appellata est enim ex uiro uirtus "le nom /virtus/ dérive du nom /virf' ;

Nat. 2, 67: Principem in sacrificando Ianum esse uoluerunt, quod ab eundo nomen est ductum... "Ils ont voulu que le premier servi dans un sacrifice fût Janus, qui tire son nom du verbe /ire/...";

Leg. 1, 60 : quae uirtus ex prouidendo est appellata prudentia "cette vertu fut appelée /prudentia/, d'après /providerel' ;

Top. 10 : Cum lex assiduo uindicem assiduum esse iubeat, locupletem iubet locupleti; is est enim assiduus, ut ait L. Aelius, appellatus ab aere dando "Quand la loi impose qu'un assidu assiste un assidu, elle veut dire qu'un riche assiste un riche : en effet, /assiduus/, comme le dit L. Aelius, vient de l'expression /aes daref';

Tusc. 3, 20 : ab inuidendo autem inuidentia recte dici potest "le terme /invidentia/ peut correctement être tiré du verbe /invideref';

Am. 100 : ex quo exardescit siue amor siue amicitia; utrumque enim dictum est ab amando ; amare autem nihil est aliud nisi eum ipsum diligere, quem ames etc. "voilà l'origine de l'ardeur de l'amour et de l'amitié ; tous deux dérivent d'/amare/; or aimer, ce n'est rien d'autre que chérir celui qu'on aime etc.".

Chez Cicéron, qui est un jusqu'au-boutiste de la correction grammaticale appliquée à l'autonymie, si le substantif autonyme est majoritairement au nominatif (comme uirtus dans l'exemple de Tusc. 2, 43 et dans presque tous / (p. 62) les exemples cités), il ne s'agit jamais d'un nominatif dénominatif et le cas est toujours justifié par la syntaxe de la phrase.

\section{Le genre et le nombre de l'autonyme : analyse d'une pratique}

Une autre différence théoriquement sensible (en pratique, peu sensible) entre les deux tendances latines à l'égard de la mention est dans le traitement syntaxique du genre et du nombre de l'autonyme. Nous avons dit qu'il devrait, en latin, être compté et accordé comme un neutre singulier. Or cette attente est très loin d'être systématiquement satisfaite.

3.1. l'autonyme a un genre et un nombre indécidables 
D'abord, en effet, il arrive bien souvent qu'on ne puisse pas décider : une ellipse ou le mécanisme de l'apposition empêche l'attribution spécifique d'un genre au segment autonymique ; l'amalgame sémiotique, déjà évoqué, a également pour conséquence de brouiller les cartes.

\subsection{1. énoncé elliptique}

De fait, les spécialistes latins du métalangage ont tendance à manier des énoncés techniques elliptiques dans lesquels il est impossible de dire si l'autonyme est neutralisé en genre et en nombre. Un cas spectaculaire d'ellipse ininterprétable sur ce point est constitué par les énoncés d'ouvrages comme l'Appendix Probi, où sont mises en parallèle une forme correcte et une forme fautive, selon le stéréotype auris non oricla " $<$ il faut dire $>$ auris, et non pas oricla". Comment savoir si les formes au nominatif ont une justification casuelle (où auris occuperait dans l'énoncé développé la fonction sujet) ou non (oportet dicere /auris/) ? Dans la première hypothèse, comment savoir s'il faut accorder l'éventuel adjectif verbal au neutre, selon les attentes modernes (/auris/ dicendum est), ou au féminin (/auris/ dicenda), selon la tendance intégrationniste ? Dès que le contexte éclaire le statut de mention du segment autonyme, l'ellipse est fréquentissime. Ainsi, si l'on traite d'étymologie, le stéréotype naturel est un schéma $X$ ductum est ou dictum est $a b Y$; il suffit alors d'indiquer de part et d'autre de la préposition ablative le terme et la source, sans le verbe facile à suppléer : $X a b Y$. Dans cette situation (Amor ab amare), comment savoir si $X$ (ici l'autonyme amor) est compté comme un neutre ou comme le masculin qu'il est dans les énoncés en usage ?

\subsection{2. apposition d'un mot métalinguistique}

L'hésitation peut provenir aussi de la présence fréquente dans la phrase métalinguistique d'un terme générique qui peut justifier à lui seul l'accord syntaxique. Un mot métalinguistique (et surtout les neutres uerbum, nomen, uocabulum) accompagne souvent l'autonyme (type Amor nomen ab amare ductum), ce qui rend impossible l'attribution d'un genre spécifique à ce dernier : / (p. 63)

Non. 53, 19 : /Parochos/ a graeco tractum est nomen... "/P./ est un nom tiré du grec";

Non. 93, 11: /Pareutactoi/ (...) a graeco uocabulum sumptum... /P./ est un terme emprunté au grec".

Le mot métalinguistique apposé à l'autonyme est suffisant, par lui-même, à entraîner l'accord au neutre de tractum ou de sumptum. On ne peut donc prouver à coup sûr que /Pareutactoi/ compte ici pour un neutre.

L'emploi d'un mot métalinguistique est notamment presque systématique à côté de mentions d'éléments non nominaux car il permet d'éluder la question du genre, du nombre (et du cas) d'un élément inerte sur ces critères : on dit couramment syllaba /in/, par exemple, car, grâce à l'apposition, tout ce segment est susceptible d'être traité comme un féminin singulier et casualisé (syllabam /in/, etc.).

QUINT. Inst. I, 6, 14 : ...genetiuos, numquam tamen eadem /ris/ syllaba terminatos "des génitifs jamais terminés par la même syllabe /ris/...": le déterminant eadem s'accorde naturellement à syllaba et cela évacue le problème du genre propre de l'autonyme /ris/.

Il en va de même avec le mot métalinguistique littera :

QUINT. Inst. I, 7, 4 : adiecta secundae syllabae s littera, "après adjonction d'un s à la deuxième syllabe": l'ajout de littera entraîne automatiquement l'accord au féminin du participe adiecta, dans cet ablatif absolu ; il devient dès lors impossible de juger du genre propre de l'autonyme $/ \mathrm{s} /$.

Par ailleurs, le mot métalinguistique concerné est si prégnant que, même si on l'omet, l'accord se fait par rapport à lui. QUINT. Inst. I, 4, 11 : atqui littera i sibi insidit (/conicit/ enim est ab illo /iacit/) et u, quo modo nunc scribitur /uulgus/ et /seruus/. Sciat etiam Ciceroni 
placuisse /aiio//Maiiam/que geminata $i$ scribere. On peut noter l'accord au féminin geminata $i$ "avec un i double" : c'est que littera $i$ de la phrase précédente est comme encore présent. Très généralement, le genre propre de littera (ou de uocalis, semiuocalis, consonans, lesquels sous-entendent littera) entraîne des accords au féminin pour les autonymes de phonèmes ou de noms de graphèmes : ISID. Et. 1, 4, 14 : pro ea $C$ et $S$ scribebant "à sa place [à la place du graphème /X/], ils écrivaient /CS/"'.

Il existe des contre-exemples au neutre :

IsID. Et. 9, 2, 58 : Philistaei ipsi sunt Palaestini, quia P litteram sermo Hebraeus non habet, sed pro eo Phi Graeco utuntur "Les Philistins sont les Palestiniens, parce que l'hébreu ne connaît pas la lettre $\mathrm{P}$ et utilise à sa place le phi grec" (notons la confusion entre phonème et graphème et, pour ce qui nous occupe surtout, la reprise de $P$ litteram par un pronom eo, sans doute neutre) ;

IsID. Et. 1, 23, 1 (à propos d'abréviations juridiques) : per unum $K$ 'caput', per duo $K K$ iuncta 'calumniae causa'... ; / (p. 64)

ISID. Et. 1, 27, 5 : Exsul addito $S$ debet scribi... "il faut écrire exsul en ajoutant un S" (mais juste après : Nam cum ipsa $X$ ex $C$ et $S$ constat...) ${ }^{32}$.

Ces neutres peuvent se justifier en soi et pour soi (genre neutralisé pour un segment autonyme, selon la méthode métalinguistique) ou être pré-déterminés par le genre du mot métalinguistique elementum qui, en concurrence avec le mot littera, peut parfois mentalement se substituer à lui.

\subsection{3. anaphore métalinguistique}

En outre, plus insidieusement, et sûrement aussi plus fréquemment, l'autonyme peut être l'objet d'une anaphore, qu'on appelle alors anaphore métalinguistique ${ }^{33}$, où le segment auto-référentiel, sans être présent physiquement, est représenté par un pronom ou un déictique ("C'est toi qui $l$ 'as dit", "Qu'entends-tu par là ?") ou par une ellipse pronominale ("on ne saurait mieux dire" = "mieux que de cette façon"; cf. Cic. Ac. 1, 41: Quonam alio modo diceres /kataleptòn/? "de quelle autre façon [ellipse: "que par comprehendibile"] pourraiton désigner le terme /k-/ ?").

L'anaphore métalinguistique est à la fois un pis-aller logique et un avantage syntaxique.

Sur le plan logique, elle est une sorte de scandale. Le plus souvent, en effet, l'anaphore métalinguistique est le lieu d'un amalgame sémiotique ${ }^{34}$, cumul entre la mention et l'usage. L'adverbe ou le pronom anaphorique utilisé dans ces tours a un statut d'autonyme et représente son référent de manière parcellaire. Ce référent, en effet, n'est pas lui-même forcément un autonyme; ce peut être un segment ordinaire, en usage. L'anaphore ne représente de ce référent que son signe en tant que tel. Par exemple, si une relative métalinguistique constitue un commentaire incident sur le terme qui, grammaticalement, est l'antécédent du pronom relatif, le rapport institué entre l'antécédent (qui est le référent) et le pronom relatif (qui est l'anaphore métalinguistique) est faux si l'antécédent n'est pas luimême un autonyme. Si j'écris "Le mot /amour/, qui a cinq lettres, etc.", l'énoncé est conforme à la logique et, qui plus est, véridique. En effet, pour le critère de vérité, le mot /amour/ a bien cinq lettres et, pour le critère de conformité, le pronom qui représente bien son antécédent "le mot /amourf". Mais si j'écris "L'amour, qui a cinq lettres, est un beau sentiment", l'énoncé est logiquement réputé absurde, car /qui/ représente non pas "l'amour" (dans l'emploi usuel qu'il a dans cette phrase), mais "le mot /amourf'. Logiquement, il est

Après tout ces hésitations existent en français : un $S$ ou une $S$ ? Les usages fluctuants d'antan (une $S$, un $\mathrm{T}$, etc.) sont unifiés dans la pédagogie moderne. L'instituteur d'aujourd'hui enseigne des lettres qui sont au masculin.

Cf. Maillard 1972.

Cf. ci-dessus. 
autonyme, mais syntaxiquement il représente un terme qui ne l'est pas. Le pronom n'est pas dans le même niveau de (méta)langage que son antécédent, ce qui est une incongruité logique. C'est néanmoins à peu près ce qu'écrit Cicéron : Am. $26:$ / (p. 65) amor, ex quo amicitia est nominata, princeps est ad beneuolentiam coniungendam; l'antécédent de la relative, amor, a bien un référent extra-linguistique et, donc, un emploi usuel : la phrase amor princeps est ad beneuolentiam coniungendam "l'amour a la première importance pour nouer des relations de cordialité mutuelle", n'est en rien métalinguistique. Le pronom relatif, en revanche, ne représente pas "l'amour", mais "le terme /Amor/'(la relative ne veut pas dire que "l'amitié dérive de l'amour" mais, conformément au sens du verbe nominare, que "le terme /Amicitia/ dérive du terme /Amor/'). Mais du coup, en vertu de la loi de prééminence du langage sur le métalangage (exprimée très clairement par Augustin, on l'a vu), le relatif, quoique de statut autonyme, s'accorde au masculin, à son antécédent en usage. C'est une manière insidieuse (probablement inconsciente) de régler un épineux problème d'accord.

La chose peut même être encore plus radicale pour peu que l'anaphore métalinguistique soit réalisée non par un pronom (qui reste variable) mais par un adverbe. Ainsi avec cet exemple cicéronien quae ita appellemus (Fin. 3, 52) : le relatif de liaison représente le terme grec proegména, ce qui est une commodité grammaticale évidente puisque la langue remplace du grec par du latin, et l'adverbe ita représente le terme latin producta, ce qui revient à remplacer du variable (producta, productorum, productis) par de l'invariable : le problème du cas propre, mais aussi du genre et du nombre de l'autonyme producta est par là même réglé en creux. D'où la belle récurrence de tours tels que sic enim appello etc., "ainsi désigné-je X", passim chez Cicéron : sic ou ita représentent un terme, qui souvent, dans la phrase précédente, n'est lui-même pas un autonyme; mais dans le segment en cours ils le représentent en autonyme tout en restant évidemment invariables.

\section{2. l'autonyme a un genre et un nombre décidables}

Hors les situations décrites ci-dessus, qui s'apparentent à une stratégie d'évitement d'un casse-tête syntaxique, le locuteur latin prend parfois (et même souvent) le parti de se révéler métalinguiste ou intégrationniste. Signalons une fois pour toutes qu'aucun auteur antique, à ma connaissance, ne se montre exclusivement métalinguiste, alors que presque tous sont exclusivement intégrationnistes.

\subsection{1. démarcation du métalangage (tendance métalinguistique)}

La tendance métalinguistique traite l'autonyme comme un mot spécifique, dont le genre et le nombre sont neutralisés. En tant que tel, il est neutre singulier quels que soient les genre et nombre propres du mot en usage qu'il représente dans le métalangage. Quelques lexicographes pratiquent ainsi occasionnellement. Voici des exemples empruntés à Isidore de Séville (écrivain au demeurant globalement intégrationniste) :

IsID. Et. 5, 33, 13 : /Idus/ autem plerique Latinorum ab edendo dictum (dictos $\mathrm{K}$ ) putant "la plupart des Latins sont d'avis que le terme /Idus/ tient son nom du verbe ledere/..."; / (p. 66)

l'accord au singulier du participe (peut-être masculin, par ailleurs) n'est peut-être qu'une bévue sur laquelle les manuscrits ne sont pas unanimes;

IsID. Et. 1, 27, 5 : /Equus/, quod est animal, per E solam scribendum (scribendus K). /Aequus/, quod est iustus, per AE diphtonga scribendum "le mot /equus/, nom d'animal, s'écrit au moyen d'un E seul; le mot /aequus/, qui signifie iustus, s'écrit au moyen de la diphtongue AE" (peut-être peut-on construire en faisant de quod non pas un relatif mais une conjonction causale) ;

ISID. Et. 1, 29, 2-3 : ...dum quaeris utrum /trames/ masculinum sit an femininum... Item /funis/... erit masculinum; 
IsID. Et. 5, 33, 13 : /Collyria/ (neutre pluriel) Latinum sonat "le mot collyria sonne latin";

IsID. Et. 7, 1, 5 : Nam /Deus/ Graece /déos/, /phóbos/ dicitur, id est /timor/, unde tractum est /Deus/ "Car/Deus/, en grec /déos/, veut dire /phóbos/, c'est-à-dire 'crainte', d'où a été tiré le nom /Deusf';

IsID. Et. 9, 4, 31 : /Barùs/ enim dicitur/grauis/, quod est/fortis/;

IsID. Et. 9, 6, 16 : /Tius/Graecum est "Tius est un mot grec";

IsID. Et. 10, 211 : /Pernix/ a pernitendo tractum ;

etc.

3.2.2. confusion entre le langage naturel et le métalangage (tendance intégrationniste)

La tendance intégrationniste ne fait pas de différence entre l'autonyme et son homonyme en usage : c'est la syntaxe usuelle qui a cours.

\subsubsection{1. le nombre}

La confusion est particulièrement flagrante à l'égard du nombre. Ainsi dans QUINT. Inst. I, 5, 2 : /Verba/ nunc generaliter accipi uolo: nam duplex eorum intellectus est "je souhaite qu'on prenne /verba/ dans son sens général : car ce mot a deux sens..." (et non pas "je souhaite que ces termes soient pris dans un sens général car ils ont deux sens"...) : noter la reprise de l'autonyme /verba/ par l'anaphorique pluriel eorum; de même SUET. Vesp. 22, 3 : admonitus ab eo /plaustra/ potius quam /plostra/ dicenda "averti par lui qu'il fallait dire /plaustra/ et non pas /plostra/..." : noter dicenda. Ou encore IsID. Et. 10, 211 : /Patriarchae/ interpretantur /patrum principes/ "/Patriarchae/ se traduit /patrum principes/ ("premiers des pères')": noter le pluriel interpretantur ${ }^{35}$. Dans un contexte qui, il est vrai, n'offre pas d'ambiguïté, le locuteur ne parvient pas (ou ne souhaite pas parvenir, par crainte du solécisme) à faire abstraction de la forme du mot mentionné : uerba (d'ailleurs recasualisé à l'accusatif et reprenant en auto-citation le mot uerbis de la phrase précédente), plaustra, patriarchae entraînent donc des accords au pluriel ${ }^{36} . /$ (p. 67)

L'incapacité à dissocier le nombre propre de l'autonyme du nombre de la forme mentionnée se manifeste remarquablement dans le traitement qui est fait des nombres mathématiques et de leur nom. Pour les nombres utilisés dans des calculs, on peut admettre que le pluriel s'impose :

IsID. Et. 3, 6, 9: III continentur a V cum aliis II partibus suis " 3 est contenu dans 5 avec un reste de 2 " 37 .

En revanche, dès qu'il est question du nom des nombres, il ne peut s'agir que d'autonymie stricte, pour laquelle un accord au pluriel est foncièrement illogique et intégrationniste :

On a vu, dans une citation ci-dessus, qu'Augustin était plus prudent à cet éagrd : Ipsum uero /arma/ quod hic uerbum est, cum a Vergilio pronuntiatum est, dictio fuit. L'autonyme /arma/ est bien compté comme un singulier.

Cela paraît évidemment absurde aux modernes. Mais songeons que le français pratique ainsi avec les noms de titres qui sont un syntagme nominal : "Les Fleurs du Mal ont été / (p. 67) composées etc." et non pas “Les Fleurs du Mal a été composé etc.”. Cf. Rey-Debove 1997 (275-276).

Notons d'ailleurs que contre toute attente, le genre de III ou $\mathrm{V}$ en tant que valeur numérique est le masculin, à cause sans doute de l'hyperonyme numerus, comme B est féminin, à cause de littera: cela se voit par exemple de ISID. Et. 3, 6, 11 : IX ad IV dum conparati fuerint etc. "en comparant 9 à 4 ...". Le français tend à considérer les nombres mathématiques comme des autonymes invariables ("3 est contenu dans 5") et à les distinguer nettement de leur emploi linguistique usuel ("Les trois autres sont arrivés", "Trois sont présents") ; parfois l'orthographe sert à distinguer les deux types d'emplois : “j”ai quatre-vingts ans" (emploi linguistique) // "Le chiffre de mes ans a passé quatre-vingt" (Hugo : emploi numérique). 
ISID. Et. 3, 3, 1-5 : /Vnus/ a Graeco nomen trahit... /Quattuor/ uero a figura quadrata nomen sumpserunt. /Quinque/ autem non secundum naturam sed secundum placitum uoluntatis uocabulum acceperunt ab eo qui numeris nomina indidit... Dicti autem /decem/ a Graeca etymologia... Porro /uiginti/ dicti quod sint decem bis geniti, U pro B littera posita... /Centum/ uocati a cantho... "/Vnus/ vient du grec... /Quattuor/ tire son nom du carré. /Quinque/ quant à lui ne tire pas son nom de la nature mais de l'arbitraire de celui qui a octroyé leurs noms aux nombres... /Decem/ a une étymologie grecque... /Viginti/ se dit ainsi parce qu'il vaut deux foix dix, la lettre $U$ ayant remplacé B... /Centum/ vient du mot /canthus/...". On voit que seul /unus/ réclame l'accord au singulier ${ }^{38}$.

Notons que, comme pour les nombres mathématiques, ces termes autonymes sont pensés comme des masculins (d'où dicti ou uocati) : cf. la note précédente. De même dans ce passage : IsID. Et. 13, 2, 4 Vnus autem atomus est, quia insecabilis est "le nombre 1 est un atome car il est indivisible", mais contra, ISID. Et. 1, 3, 10 Alpha littera apud eos uocatur in numeris /unum/. Vbi scribunt Beta, uocatur /duo/; ubi scribunt Gamma, uocatur in ipsorum numeris /tres/; ubi scribunt Delta, uocatur in numeris ipsorum /quattuor/ "alpha, chez eux, vaut pour le nombre 1 etc." : il est notable que si /tres/ est au masculin, comme attendu, /duo/ a un genre indécidable et /unum/ est pour une fois au neutre. / (p. 69)

\subsubsection{1. le genre}

Il est inutile d'accumuler beaucoup d'exemples nouveaux. De multiples cas d'attraction de l'autonyme au genre de son homonyme en usage sont attestés dans ce qui précède. À cette moisson, ajoutons, en ouvrant presque au hasard Isidore de Séville :

ISID. Et. 5, 31, 1 : /Nox/ a nocendo dicta "/Nox/vient de /nocere/";

ISID. Et. 3, 71, 1 : /Sol/ appellatus eo quod solus appareat "/Sol/vient de ce que le soleil apparaît seul" ;

IsID. Et. 5, 26, 9 : /Falsitas/ appellata a fando aliud quam uerum est “/Falsitas/vient de /fari aliud quam uerum est/, 'dire autre chose que le vrai'", etc.

3.3. un quasi-système : le lexicographe Nonius

Nonius Marcellus, à cet égard, présente plus que sporadiquement un semblant de système. Dans son dictionnaire du latin républicain, il illustre les termes dont il parle par une ou plusieurs citation(s) d'auteur(s).

L'entrée dont il part est parfois présentée sous une forme d'étiquette, au nominatif ou à l'infinitif :

NON. 166, 7 : /Gracilitudo/ pro/gracilitas/...

Non. 478, 9 : /Ferus/significat/seuerus/...

Non. 184, 11 : /Irascere/ pro /irasci/...

On trouve aussi chez lui, avec une bonne fréquence, des énoncés intégrationnistes dans lesquels au moins un autonyme est casualisé par la préposition ou la construction :

NoN. 209, 5 : /Numen/ pro ornatu...

NON. 478, $9:$ /Ferus/ iterum significat ceruum...

NoN. 617, 4 : /Sufficere/ ab inficiendo...

Parfois, et même fréquemment, l'entrée est lemmatisée sous la forme que prend le mot expliqué dans la première illustration citée ; le deuxième autonyme est, quant à lui,

-soit au même cas que l'entrée (autonymie directe): NoN. 266, 1: /Tetrical est /seuera/... (l'adjectif est au nominatif féminin dans l'illustration citée) ;

-soit casualisé selon l'usage (autonymie indirecte, selon la méthode intégrationniste) : 
NoN. 196, 8 : /Luculentitatem/ ab luculento... (l'accusatif est dans l'illustration) ;

-soit, s'il s'agit s'une forme non nominale, à une forme identique à celle de l'entrée :

Non. 21, 25 : /Enoda/ significat lexplanal... (suivent plusieurs exemples, dont le premier comporte effectivement l'impératif enoda); NoN. 32, 18: /Gliscit/ est /congelascit/ et /colligitur/ uel /crescit/ uel /ignescit/ (en l'occurrence, la forme gliscit n'est pas le lemme utilisé dans les illustrations, mais ses synonymes ont tous la même analyse formelle). / (p. 69)

Mais, et voilà qui nous paraît relever d'une réflexion au moins partielle sur le statut spécial de l'autonyme, Nonius fait un sort spécial aux entrées adjectivales : ces dernières sont

-soit lemmatisées comme n'importe quelle autre partie du discours, comme ci-dessus tetrica : Non. 253, 16 : /Squalam/ pro /squalidam/... (le lemme de l'illustration est squalam); Non. 256, 19 : /Scelerosi/pro /scelerati/... (le lemme de l'illustration est scelerosi),

-soit mis sous une forme d'étiquette au nominatif singulier (comme c'est souvent le cas avec les substantifs) : NoN. 147, 8 :/Errabundus/pro /errans/(le lemme de l'illustration est le neutre errabunda [uestigia]); NoN. 508, 12: /Inpurus/ est /aliquo uitio maculatus/... (le lemme de l'illustration est l'ablatif impura) ; NoN. 556, 4 : /Nobilis/ est /notus/... (le lemme de l'illustration est nobilitata); NoN. 643, 23 : /Stupidus/ est /obstupefactus/... (le lemme de l'illustration est stupidissimo); etc.

Mais alors, on constate que ce nominatif est majoritairement au neutre, quelle que soit la forme que prend l'adjectif dans les illustrations citées par Nonius: NoN. 258, 5 : /Subsiciuum/, /secunduum/, /sequens/" <l'adjectif>/subsisicuus/ <a le sens de>/secundus/, /sequensf' (suit un exemple qui utilise le nominatif féminin subsiciua [opera]) ; Non. 269, 7 : /Verecundum/, /audax et confidens/ " $<$ l'adjectif> /verecundus/ <équivaut à> /audax et confidensf' (suit un exemple qui utilise la forme uerecunda) ; Non. 170, 4 : /Generosum/, /nobile/ uel /forte/ (suit un exemple qui utilise la forme generoso) ; NoN. 188, 19 : /Inmissum/ pro /demissum/ et /longius pendens/; NoN. 36, 22 : /Nalgum/ est proprie /intortum/ (suit un exemple qui utilise la forme ualgus); etc.

Plus généralement, des phénomènes d'accord d'un participe, dans des énoncés moins elliptiques, ou d'un adjectif, montrent avec une fréquence notable, mais sans qu'il y ait un système strict, que l'autonyme principal (l'entrée) est traité comme un neutre singulier (le deuxième autonyme est parfois accordé à la mode intégrationniste). Si cela n'est pas étonnant lorsque l'entrée est un infinitif ou un substantif neutre singulier :

Non. 246, 5: /Recentari/ honeste positum pro /nouari/ "/Recentari/ est correct pour remplacer /novarif';

NON. 177, 23 : /Inplicare/ positum pro /ornare/;

Non. 270, 18 :/Viscus/positum pro /uiscera/ "/Viscus/ est mis pour /visceraf', cela paraît l'être davantage quand l'entrée est un mot doté d'un genre et d'un nombre spécifiques :

Non. 255, 10 : /Subiti/dictum pro /repentini/ "/Subiti/ est utilisé pour /Repentini/P' (les autonymes sont lemmatisés, mais l'accord de dictum est notable) ;

Non. 266, 15 : /Tenta/dictum pro /extensa/ (lemmes au neutre pluriel) ;

Non. 271, 4 :/Nagor/dictum pro/uagitus/; / (p. 70)

Non. 189, 7 : /Iactuosae/ positum pro /iactantes/;

Non. 207, 10 : /Multitudo/positum pro /frequentia/ aut/copia/;

NoN. 207, 19 : /Memoria/ positum pro /uetustate/;

NoN. 207, 4 : /Ministratrix/positum pro /ministra/;

NoN. 103, 5 : /Adsestrix/femininum est ;

NoN. 55, 17 :/Idiotas/ a graeco tractum, /inutilis/;

etc.

Avec ces derniers exemples (qui représentent un type très fréquent chez Nonius), on est de plain-pied dans la méthode des lexicographes modernes. 


\section{CONCLUSION :}

La question du genre et du nombre des autonymes en latin ne se pose pas de manière théorique chez les auteurs antiques, et le chercheur moderne qui s'interroge a posteriori sur ce problème ne trouvera chez les auteurs de la période latine que des réponses pratiques. Saint Augustin lui-même, dont on a rappelé quelques idées essentielles à l'égard du métalangage, reste bloqué dans son analyse avant de formuler une quelconque règle syntaxique sur les accords qu'engagent les autonymes de noms communs.

Ce qui le bloque sans doute, c'est la nature de la langue latine, dans laquelle cas, genre et nombre induisent des phénomènes d'accord très prégnants. Et bien sûr, l'obstacle majeur reste que ces réflexions d'Augustin se formulent en latin et qu'il ne peut établir des règles de syntaxe qui contredisent celles qui ont cours en langue : il ne parvient pas à distinguer le latin et le méta-latin. Les latinophones natifs semblent dépourvus de toute capacité à analyser le latin comme un objet extérieur. La casualité et l'absence d'article sont pour la plupart d'entre eux d'indéniables freins à la prise en compte d'un statut spécial des mots en mention.

$\mathrm{Au}$ Moyen-Âge, la question de l'autonymie va se poser de manière très technique : la logique médiévale développe les oppositions de nom et de chose et la notion de suppositio materialis ; l'autonymie devient un carrefour, un passage obligé vers les syllogismes et vers la grammaire ${ }^{39}$. On peut estimer, avec I. Rosier (2003), que ce retour efficace sur le latin s'explique par l'émergence de nouvelles langues vernaculaires; le latin, tout en restant le véhicule de la culture et de la connaissance internationales, est devenu langue étrangère ; à ce titre, il est revisité par des locuteurs qui parlent des langues éventuellement non casuelles et éventuellement dotées de l'article ${ }^{40}$. C'est cette circonstance historique qui a permis des progrès décisifs en dialectique. / (p. 71)

\section{Références :}

Augustin, De dialectica (387) : édition électronique de James Marchand, Univ. of Illinois, sur http://ccat.sas.upenn.edu/jod/texts/dialectica.html. Traduction seule dans Baratin \& Desbordes 1981, p. 211-231 (texte 45).

Augustin, De magistro (389) : dans la Patrologie latine de Migne, tome 32 ; en outre, édition électronique www.augustinus.it/latino/maestro/maestro.htm. Traduction seule des $\S 20$ et 22-38 dans Baratin \& Desbordes 1981, p. 232-246 (texte 46).

Auroux (coord.) : Sylvain Auroux (sous la direction de), Histoire des théories linguistiques, 3 tomes (1989, 1992, 2000), Mardaga, Bruxelles.

Authier-Revuz 1992 et 1993 : Jacqueline Authier-Revuz, "Repères dans le champ du discours rapporté ", L'Information Grammaticale, 55 (Octobre 1992), 38-42, et 56 (Janvier 1993), 10-15.

Baratin 1989 a : Marc Baratin, “La constitution de la grammaire et de la dialectique ”, dans Auroux (coord.), tome 1, 186-206.

Baratin 1989 b : Marc Baratin, “La maturation des analyses grammaticales et dialectiques ”, dans Auroux (coord.), tome 1, 207-227.

Baratin et Desbordes 1981: Marc Baratin et Françoise Desbordes, L'analyse linguistique dans l'Antiquité classique, Klincksieck.

Desbordes 1988: Françoise Desbordes, “Homonymie et synonymie d'après les textes théoriques latins ", dans L'Ambiguïté (I. Rosier éd.), Presses universitaires de Lille, 51-102. 
Festus : Sextus Pompeius Festus De verborum significatu quae supersunt cum Pauli Epitome, edidit W. M. Lindsay, Teubner, Leipzig, 1913 (reprint 1965 chez Olms, Hildesheim).

Isodore de Séville: Isidori Hispalensis Episcopi Etymologiarum sive Originum libri XX, edidit W. M. Lindsay (3 vol.), Oxford Classical Texts, 1911 (reprint non daté).

Libera \& Rosier (1992): Alain de Libera et Irène Rosier, "La pensée linguistique médiévale", dans Auroux (coord.), tome 2, 115-186.

Maillard 1972 : Michel Maillard, “Anaphores et cataphores ”, Communications, 19, 93-104.

Nicolas 2000 : Christian Nicolas, " Autonymie et autonomie syntaxique : le latin confronté au métalangage ”, Revue de Philologie 74-1 (1999), Klincksieck, Paris, 43-68.

Nicolas 2001 : Christian Nicolas, "Syntaxe de la mention : l'exemple de Cicéron ", De lingua latina novae quaestiones, Actes du $X^{e}$ Colloque International de Linguistique Latine (C. Moussy éd.), BEC, Peeters, Louvain-Paris, 497-509.

Nicolas 2003 : Christian Nicolas, “Typologie de l'autonyme en latin ”, à paraître dans les Mélanges Catherine Dobias.

Nonius Marcellus : De compendiosa doctrina, edidit W. M. Lindsay (3 vol.), Teubner, Leipzig, 1903 (reprint 1964 chez Olms, Hildesheim).

Recanati 1979 : François Recanati, La transparence et l'énonciation, Seuil.

Rey-Debove 1997 : Josette Rey-Debove, Le métalangage, Armand Colin.

Rosier 2003 : Irène Rosier-Catach, " La suppositio materialis et la question de l'autonymie au Moyen Âge", intervention au colloque "Le fait autonymique" (Paris III, automne 2000), publié en ligne (sur www.cavi.univ-paris3.fr/ilpga/autonymie/actes.htm). / (p. 72)

Serbat 1996: Guy Serbat, L'emploi des cas en latin (= Grammaire fondamentale du latin, tome VI). Vol. I : Nominatif, Vocatif, Accusatif, Génitif, Datif, BEC, Peeters. 